\title{
Study on Grid Scheduling Model Based on Hierarchical Scheduling Model
}

\author{
Liu Feng ${ }^{1}$ and Guo Weiwei ${ }^{2}$ \\ ${ }^{1,2}$ Heilongjiang University of Technology \\ JiXi 158100, china \\ ${ }^{1}$ Liufeng8038@163.com, ${ }^{2}$ gwwguoweiwei@163.com
}

\begin{abstract}
In order to solve the task allocation and scheduling problem based on the analysis and research of hierarchical Petri net, this paper proposed a grid scheduling model based on hierarchical scheduling model. This model can ensure that the various elements of the loose coupling between the scheduling, to facilitate data resources deployment in grid environment; in addition, this paper proposed thought of the variable structure Petri network, through this technology can dynamic adjust work modeling and resource allocation, and to support the mission of exception handling; finally, to verify the rationality of the scheduling algorithm, and analyzes the performance and the efficiency of resource allocation algorithm.
\end{abstract}

Keywords: Data mining, Petri nets, Grid scheduling, Job scheduling model

\section{Introduction}

As everyone knows, the need of data mining from known disorder, random, mass, even huge data resources in a higher dimensional find important information and knowledge to people [1-2]. For the telecom enterprises and other large data resources service providers and managers, the application of data mining is very important. However, as data volumes grow indefinitely, straight up the application of complexity, the development of data mining technology is to face this challenge, by means of cluster computing or improved single algorithm to improve the performance of data mining, which has been unrealistic. Therefore, data mining grid emerge is as the times require by combining the grid technology with data mining technology, forming a new data mining grid system [3-4].

In the data mining grid system, after task scheduling of each data mining subjects, resource is assigned to tasks within the dependence of indivisible. In order to reasonably allocate resources and make good use of these relationships, to ensure the successful implementation of the mining task, it must solve good job allocation and scheduling problem. In data mining grid, the scheduling refers to the process of data mining [5-6], the system required for each behavior, the error of single point failure recovery, and provide fault tolerance mechanism is powerful, for some runtime error give a reasonable solution data mining tasks, to ensure the smooth, let the customers satisfy with the result of data mining [7-8].

\section{Extended Definition of Petri Net}

Firstly, in this paper, Petri network structure of the traditional variable structure is adjusted puts forward the idea of. This new Petri network can accurately define the job scheduling and task assignment and other work, and the accuracy and rationality of the whole data mining process. The so-called operation is based on granularity, the scheduling layer decomposition; the overall scheduling process of upper is focus on jobs, lower pay attention to people the content of specific operation. Petri network with the hierarchical design, scheduling model build, which can reduce the distributed scheduling and computation overhead is effectively, 
improve the accuracy and efficiency of data mining grid system [9].

At present, research methods of job scheduling are in two kinds [10]: one kind is called a script based on approach, and the other is called a graph based on approach. This method belongs to the latter. The former is relatively simple, but because the script contains grammatical information, so the analysis and understanding of the grammar will spend a lot of time; and graph is based on methods, its greatest advantage is clear, Petri net belongs to this kind of method. It is a dynamic, it can be used in parallel or asynchronous system modeling tools, the use of simple expressed in various forms, are widely used in a variety of data analysis [11].

In order to meet the needs of data mining in grid system, this paper makes some extensions to the Petri network system: (1) in order to solve the scheduling problem, put forward the layered idea, the scheduling of tasks are from top to bottom, decomposition, reducing the complexity of scheduling activities; (2) in order to adapt to the decomposition of the operation, realize the dynamic adjustment of tasks, and the abnormal task for the timely processing, put forward the concept of variable structure.

Definition: layer with colored Petri net belongs to a group of 9 tuple: PETRI $=\{Q, R, F, D, C, I, O, K, M\}, Q$ is a non-empty set, store the contents of the current system. $R$ is a collection of non-empty change. $F$ is the arc set, used to determine the dispatching operation of all kinds of input and output. $D$ is a color set. $C$ is a coloring function. $I$ and $O$ are the function changes of $R, I$ refers to the input function, $O$ refers to the output function. $K$ is the accommodation the number function of $R . M$ called grid initial signs. In the variable structure of the colored Petri net, each node and operation need to view the change results of each other, and use the indirect intermediate values to guide the operation state own and task execution

\section{Design and Implementation of the Job Scheduling Model}

\subsection{Hierarchical Scheduling System}

According to the construction characteristics of grid data mining system, put forward the scheduling model based on the hierarchy of structure, and combined with the characteristics of Petri network variable, design the operation model of the corresponding. Three layer networks for distributed mining operations are managed, defines the basic strategy of job allocation and scheduling.

\subsubsection{The First Layer: Job Scheduling Network}

Scheduling network is responsible for the management job operation, including job queue management and operation condition monitoring. This paper defines four kinds of operation state, including: wait, operation, and state failure

(1) Wait state: selected work was submitted, but not yet started;

(2) The operation state: running, no obstruction;

(3) Completed state: all independent operations or contain sub operation completed;

(4) Failed state: operation or including sub operation failed;

Job scheduling is built based on PETRI network structure, system architecture is shown in Figure 1. 


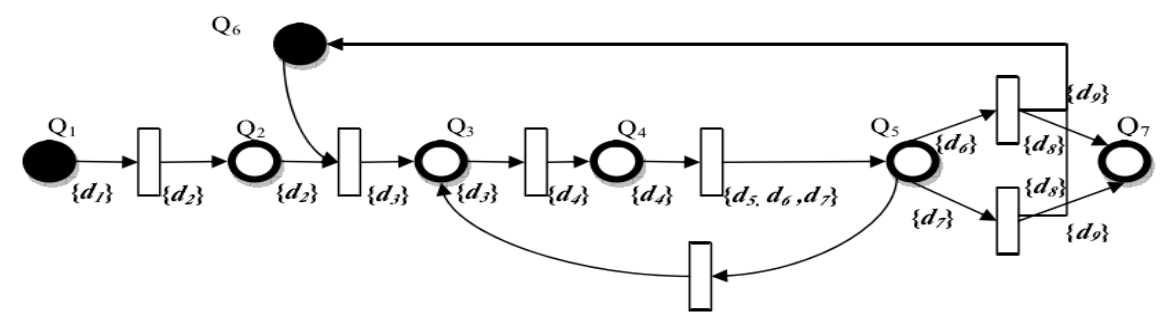

Figure 1. Task Scheduling Networks

It can be seen from Figure1. $\mathrm{Q}=\left\{\mathrm{q}_{i} \mid 1 \leq \mathrm{i} \leq 7\right\}, \mathrm{R}=\left\{\mathrm{r}_{i} \mid 1 \leq i \leq 7\right\}$, A detailed description is as shown in Table 1.

Table 1. Descriptions of Task Scheduling and Changes

\begin{tabular}{|c|c|c|c|}
\hline Library number & Description & Change number & Description \\
\hline 1 & Submit job Library & 1 & Select the operation \\
\hline 2 & $\begin{array}{c}\text { Wait for the job } \\
\text { Library }\end{array}$ & 2 & Start running \\
\hline 3 & Running jobs & 3 & Select Check \\
\hline 4 & $\begin{array}{l}\text { To check the } \\
\text { homework }\end{array}$ & 4 & Check status \\
\hline 5 & Check operation & 5 & Labeling operations \\
\hline 6 & $\begin{array}{l}\text { The amount of work } \\
\text { restrictions }\end{array}$ & 6 & Successful feedback \\
\hline 7 & Quit the job Library & 7 & Failure to return \\
\hline
\end{tabular}

\subsubsection{Second Layer: Job Scheduling Network}

The lower sub job scheduling is in job scheduling network, is mainly responsible for the management of sub job execution, system architecture is shown in Figure 2. It is colored net a hierarchical design, it is detailed as follows:

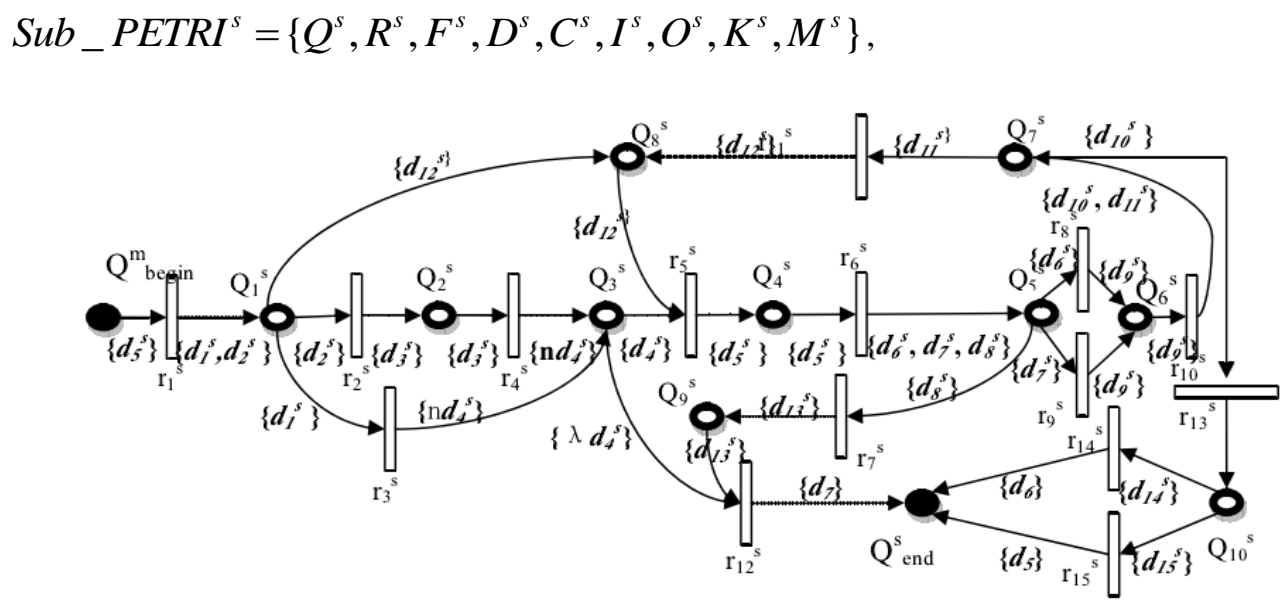

Figure 2. Sub_Task Scheduling Networks

It can be seen from Figure 1. $Q^{s}=\left(q_{\text {begin }}^{s}, q_{\text {end }}^{s}, q_{i}^{s} \mid 1 \leq i \leq 10\right\}, R^{s}=\left\{r_{i}^{s} \mid 1 \leq i \leq 15\right\}$, A detailed description is as shown in Table2 


\subsubsection{Third Layer: Task Scheduling Network}

Task scheduling is a scheduling network under a layer, Figure 3 complex changes of the $r_{6}^{s}$ decomposition of the third sub-network. System architecture is shown in Figure3.Task scheduling network data is describing the formula is as follows:

$$
\text { M_PETRI }=\left\{Q_{m}, R_{m}, F_{m}, D_{m}, C_{m}, I_{m}, O_{m}, K_{m}, M_{m}\right\}
$$

Table 2. Descriptions of Sub_Task Scheduling and Changes

\begin{tabular}{|c|c|c|c|}
\hline No. & Description & Changes & Description \\
\hline Begin & $\begin{array}{l}\text { Start sub Job } \\
\text { Network }\end{array}$ & 1 & $\begin{array}{c}\text { Check the } \\
\text { initialization state }\end{array}$ \\
\hline End & $\begin{array}{l}\text { End Sub Job } \\
\text { Network }\end{array}$ & 2 & $\begin{array}{c}\text { Analysis of } \\
\text { operation network }\end{array}$ \\
\hline 1 & Initial inspection & 3 & $\begin{array}{l}\text { Extraction of a sub } \\
\text { job }\end{array}$ \\
\hline 2 & $\begin{array}{l}\text { The sub working } \\
\text { Gallery }\end{array}$ & 4 & Promoter activity \\
\hline 3 & Running the subjob & 5 & $\begin{array}{l}\begin{array}{r}\text { Selection of } \\
\text { scheduling }\end{array} \\
\end{array}$ \\
\hline 4 & To schedule job & 6 & $\begin{array}{l}\text { Scheduling sub } \\
\text { operation }\end{array}$ \\
\hline 5 & Sub jobs scheduled & 7 & $\begin{array}{l}\text { Remove the } \\
\text { markings }\end{array}$ \\
\hline 6 & $\begin{array}{l}\text { The job has been } \\
\text { marked }\end{array}$ & 8 & $\begin{array}{l}\text { Sub normal } \\
\text { operation }\end{array}$ \\
\hline 7 & Job status check & 9 & The sub task \\
\hline 8 & $\begin{array}{l}\text { The sub task } \\
\text { selection }\end{array}$ & 10 & $\begin{array}{l}\text { Check whether the } \\
\text { scheduling. }\end{array}$ \\
\hline 9 & Remove operation & 11 & Job selection marker \\
\hline 10 & $\begin{array}{l}\text { Check the operating } \\
\text { states }\end{array}$ & 12 & Operation failed \\
\hline & & 13 & Complete check \\
\hline & & 14 & Finish marking \\
\hline & & 15 & $\begin{array}{c}\text { The normal } \\
\text { operation of marker }\end{array}$ \\
\hline
\end{tabular}




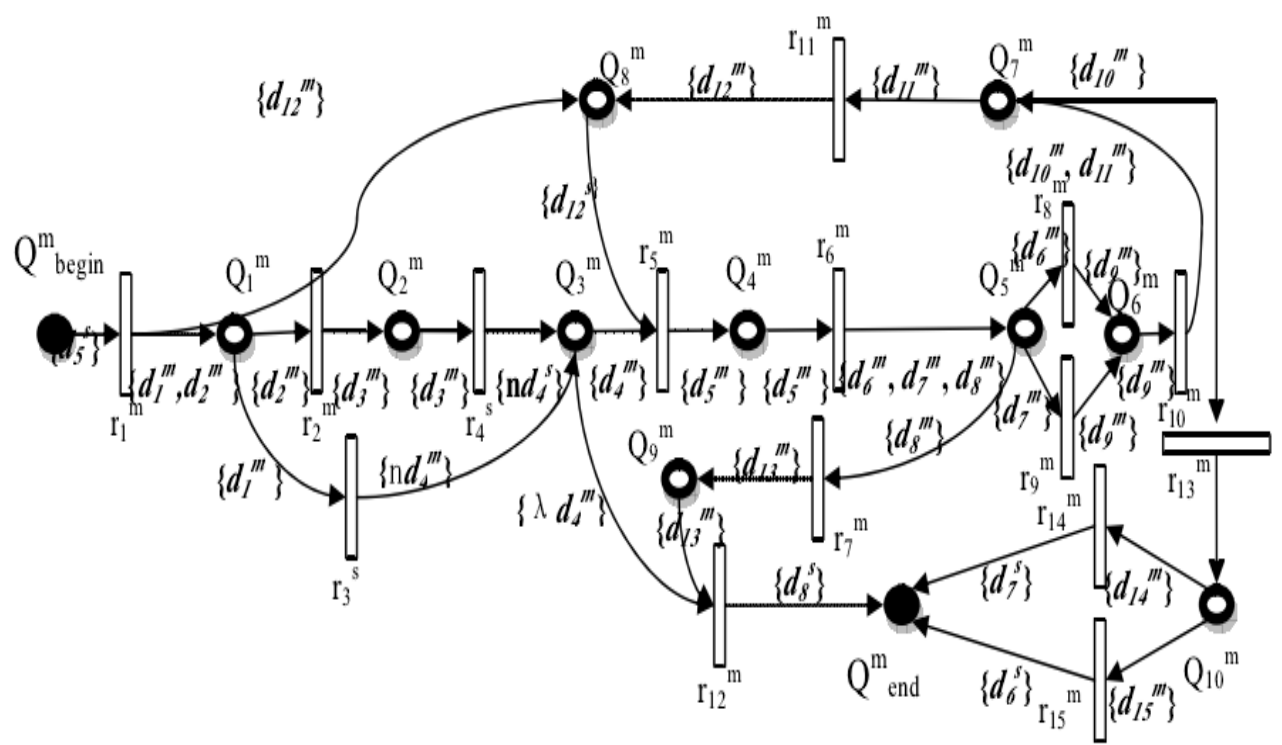

Figure 3. Mission Scheduling Networks

It can be seen from Figure $1 . Q^{m}=\left(q_{\text {begin }}^{m}, q_{\text {end }}^{m}, q_{i}^{m} \mid 1 \leq i \leq 10\right\}, R^{m}=\left\{r_{i}^{m} \mid 1 \leq i \leq 15\right\}$, A detailed description is as shown in Table 3

Table 3. Descriptions of Mission Scheduling and Changes

\begin{tabular}{|c|c|c|c|}
\hline No. & Description & Changes & Description \\
\hline Begin & Sub network & 1 & $\begin{array}{c}\text { Check the } \\
\text { initialization state }\end{array}$ \\
\hline End & End sub network & 2 & Switching network \\
\hline 1 & $\begin{array}{l}\text { Task initialization } \\
\text { check }\end{array}$ & 3 & The extraction task \\
\hline 2 & The task graph & 4 & Start the task \\
\hline 3 & Running the task & 5 & $\begin{array}{l}\text { Select the task } \\
\text { scheduling }\end{array}$ \\
\hline 4 & The scheduling task & 6 & The scheduling task \\
\hline 5 & The scheduled task & 7 & Task clear marks \\
\hline 6 & The marked tasks & 8 & The normal work \\
\hline 7 & $\begin{array}{l}\text { Task state } \\
\text { examination }\end{array}$ & 9 & Task completion \\
\hline 8 & Task selection & 10 & $\begin{array}{l}\text { Check whether the } \\
\text { scheduling. }\end{array}$ \\
\hline 9 & Task clear operation & 11 & $\begin{array}{l}\text { Task selection } \\
\text { marker }\end{array}$ \\
\hline 10 & $\begin{array}{l}\text { Check the operating } \\
\text { states }\end{array}$ & 12 & $\begin{array}{l}\text { The failure of the } \\
\text { mission }\end{array}$ \\
\hline & & 13 & Complete check \\
\hline & & 14 & $\begin{array}{l}\text { Task completion } \\
\text { marks }\end{array}$ \\
\hline & & 15 & $\begin{array}{l}\text { Normal operation } \\
\text { marking }\end{array}$ \\
\hline
\end{tabular}




\subsection{Parallel Operation Network}

This paper presents work mode of operation net, it is mapped to a workflow, job network is managed by the upper hierarchical network, by scheduling network application of decomposition, according to the user to adjust the operation network. This paper presents a flexible structure Petri network.

According to the operation granularity, job network can be subdivided into sub job network, task and sub task network.

\subsubsection{Sub Job Network}

Sub job has exactly the same structure with homework and independent operation flow. The job has its independent data input, output interface and operation of a complete set of rules. Sub job network was obtained by analyzing the job description file, the definition is

$$
C S=\left\{Q^{1}, R^{1}, F^{1}, D^{1}, C^{1}, I^{1}, O^{1}, \Phi^{1}, \Psi^{1}, M^{1}, M^{0}\right\}
$$

$Q^{1}$ is input, output sub task set; $R^{1}$ mainly includes four kinds of operations: exclusive transmission type sub operation, exclusive computational sub jobs, sharing transmission type sub operations, and shared computing sub operation; $D^{1}$ including external remote data, local data, internal external and internal local data and remote data; $\Phi^{1}, \Psi^{1}$ represents the overhead time consumption and system operation.

\subsubsection{Task}

Produced by the decomposition of sub-jobs, a sub operation can contain one or more tasks, the definition is

$$
C M=\left\{Q^{2}, R^{2}, F^{2}, D^{2}, C^{2}, I^{2}, O^{2}, \Phi^{2}, \Psi^{2}, M^{2}, M^{1}\right\}
$$

$Q^{2}$ is input, output task set; $R^{2}$ contains five kinds of task types, internal data, external data transmission, data partitioning parallel computing, not parallel computing, parallel computing and indivisible. $D^{2}$ includes five kinds of data types, namely the external remote data, remote data, local data, internal external local data, and computing resource data. $\Phi^{2}, \Psi^{2}$ represents the overhead time consumption and system task

\subsubsection{Sub Tasks}

For the task scheduling network, it can be decomposed to single task and further, we can get a sub task network. An independent tasks can be dynamically decomposes into 1 or more sub task. The definition is

$$
C M=\left\{Q^{3}, R^{3}, F^{3}, D^{3}, C^{3}, I^{3}, O^{3}, \Phi^{3}, \Psi^{3}, M^{3}, M^{2}\right\}
$$

$Q^{3}$ is input, output sub task set; $R^{3}$ mainly consists of four subtasks, namely exclusive type transmission sub task, exclusive type sharing transmission sub task subtask,, and shared computing tasks; $D^{3}$ includes five kinds of data types, namely the external remote data, remote data, local data, internal external local data, and computing resource data. $\Phi^{3}, \Psi^{3}$ represents the overhead time consumption and system task. 


\section{Experiment Design and Discussion}

In this paper, in order to demonstrate the accuracy of grid scheduling model. Need to be structural optimization analysis and experimental verification. The time complexity and overhead using transition tree analysis, in addition to research grouping task.

\subsection{Structure Optimization Analysis}

For the grid scheduling model, Parallel computing tasks require strict accordance with the requirements of data transmission functions and operational characteristics and task decomposition. The decomposition has to maintain data consistency is one of the most important principles. Decomposition is by operation to task, and the task is to the two sub tasks, to ensure that the data content of all treatment remained unchanged in the merging process pretreatment, transfer and block.

In the data mining grid system, in order to achieve better performance of parallel computing, and to unify the grid scheduling and grid work, based on the proposed parallel computing task was optimized structure, defined as follows:

If the remote data input sub job $t_{1}^{i}$ has $\mathrm{k}$, at this time, the system will calculate the task decomposition into $l$. It is as shown in Figure4.

By increasing the amount of change and crossing nodes of the way, make network reduce the $l$ task, the corresponding theory of time and task cost are greatly reduced. Suppose $l$ task changed is $\left\{t^{r} \mid 1 \leq r \leq l\right\}$. Then reduce the overhead is $\sum_{r=1}^{l} \Phi_{r}\left(t^{r}\right)$.

\subsection{Comparative Analysis of Costs}

This algorithm (T1) and Petri net grid scheduling model presented in Literature [12] (T2) are compared and analyzed, the analysis are divided into two stages.

The first stage: the algorithm only applies scheduling network, without adding concurrent job network model, and the results of T2 data:

Assuming the grid now has 3 tasks and 3 computing resources, running time and expense ratio are as shown in Table 4:

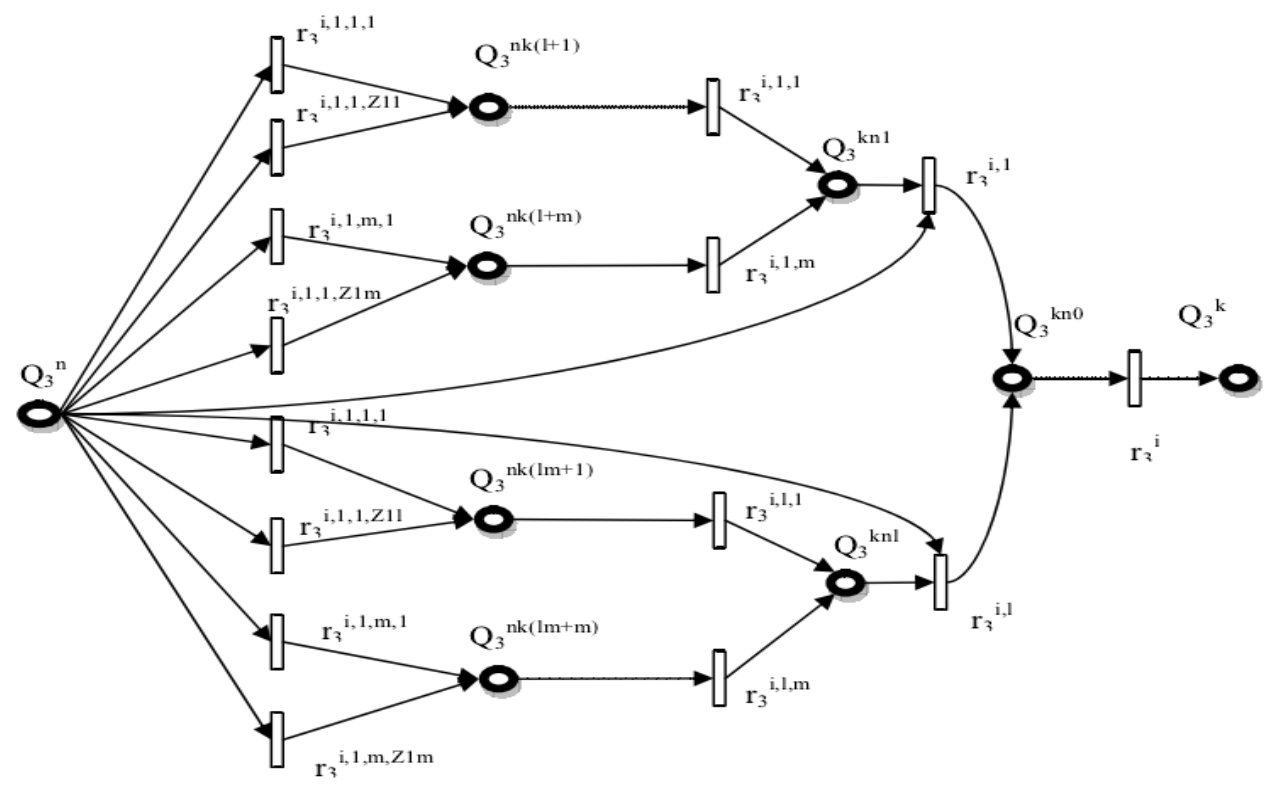

Figure 4. Diagram of Parallel Computing Task Structural Optimized 
Table 4. Descriptions of Task Running Time and Expense Ratio

\begin{tabular}{|l|c|c|c|c|c|c|}
\hline $\begin{array}{c}\text { TComputing } \\
\text { task }\end{array}$ & $\begin{array}{c}\text { EExecution } \\
\text { time } \\
\text { (T1\&T2) }\end{array}$ & $\begin{array}{c}\text { TTransmission } \\
\text { time } \\
\text { (T1\&T2) }\end{array}$ & $\begin{array}{c}\text { Estimated cost } \\
\text { (T1\&T2) }\end{array}$ & TCost cap & $\begin{array}{c}\text { TTime } \\
\text { limit }\end{array}$ & RRatio \\
\hline 1 & $5 \& 6$ & $3 \& 7$ & $10 \& 9$ & 18 & 13 & $1: 2$ \\
\hline 2 & $2 \& 3$ & $2 \& 2$ & $4 \& 4.5$ & 5 & 6 & $1: 3$ \\
\hline 3 & $4 \& 10$ & $3 \& 9$ & $8 \& 15$ & 10 & 14 & $1: 4$ \\
\hline
\end{tabular}

The results can be seen from Table 4, compared this paper algorithm with the T2 algorithm, which requires less time and cost, better than the T2 algorithm. But the two kinds of algorithm in task execution process have obvious characteristics of load imbalance.

The second stage: adding a complete algorithm concurrent job network, following with the comparative results of data $\mathrm{T} 2$ :

Assuming the grid now has 4 tasks and 2 computing resources, running time and expense ratio are as shown in Table 5:

Table 5. Descriptions of Task Running Time and Expense Ratio

\begin{tabular}{|c|c|c|c|c|c|c|}
\hline $\begin{array}{c}\text { TComputing } \\
\text { task }\end{array}$ & $\begin{array}{c}\text { EExecution } \\
\text { time } \\
(\text { T1\&T2) }\end{array}$ & $\begin{array}{c}\text { TTransmission } \\
\text { time } \\
\text { (T1\&T2) }\end{array}$ & $\begin{array}{c}\text { Estimated cost } \\
\text { (T1\&T2) }\end{array}$ & TCost cap & $\begin{array}{c}\text { TTime } \\
\text { limit }\end{array}$ & RRatio \\
\hline 1 & $5 \& 6$ & $3 \& 7$ & $10 \& 9$ & 18 & 13 & $1: 2$ \\
\hline 2 & $2 \& 3$ & $2 \& 2$ & $4 \& 4.5$ & 5 & 6 & $1: 3$ \\
\hline 3 & $4 \& 10$ & $3 \& 9$ & $8 \& 15$ & 10 & 14 & $1: 4$ \\
\hline 4 & $6 \& 9$ & $4 \& 8$ & $13 \& 12$ & 13 & 15 & $1: 3$ \\
\hline
\end{tabular}

The results can be seen from Table5, compared the paper algorithm with T2 algorithm, this paper algorithm strategy execution time and objective function value are better than not join the concurrent job network. The whole algorithm is better than T2, it can achieve better load balancing.

\subsection{Model Performance Analysis}

In order to grid scheduling model and operation management function is the basic performance verification, this paper uses the stochastic simulation method to structural task request, and then examine the allocation of resources and tasks in the model, according to the data structure, From running time of tasks, scheduling time and task planning carried on the analysis. In the data comparison, the selected object is still Petri net grid scheduling model presented in Literature [12].

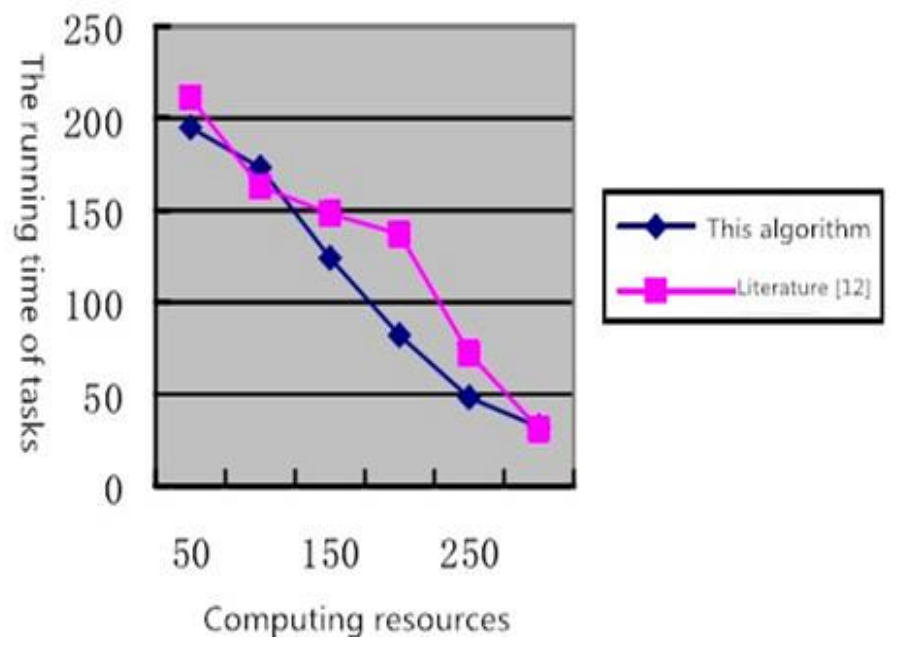

Figure 5. Diagram of Relations Between Resources and Mission Running 
In the Figure 5, we can draw the following content, due to increasing number of computing resources, the number of nodes to assume the task of computing will increase. With the equilibrium allocation of tasks, the task running time is reduced, both the scheme proposed in this paper and Literature [12] proposed schemes are obviously.

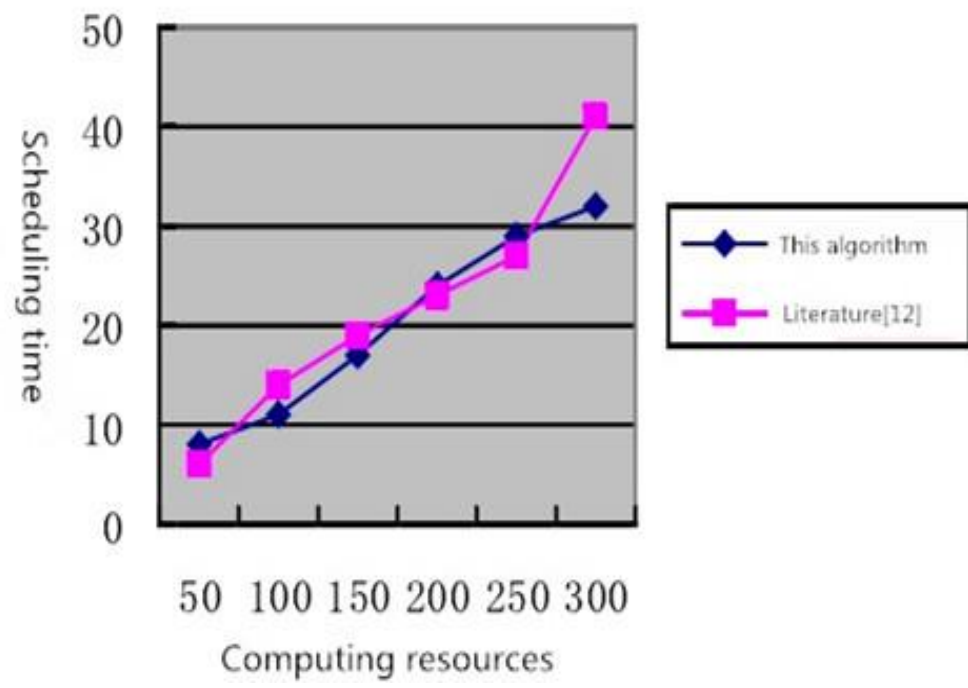

Figure 6. Diagram of Relations Between Resources and Scheduling

In the Figure 6, we can draw the following content, when computing resources quantity increasing, although the number of nodes increases, but the node scheduling complexity increase.

\section{Conclusion}

This paper presents operation model and scheduling model based on variable structure. Based on the hierarchical scheduling network, it is convenient for scheduling and parallel processing on different granularity of operations. In addition, the variable structure strategy was proposed, according to the actual resource allocation adjustments for task execution status and specific content and with fault tolerance and fault handling mechanism. Finally, the correctness of reachability tree model of job network structure is analyzed and validated, analyzing the time characteristics of job network and discussion of resource allocation optimization strategy.

\section{References}

[1] L. Weidong, S. Jiaxing and L. Chuang, "Calculation and analysis model of computing grid price based on time Petri net”, Acta electronica Sinica, vol. 33, no. 8. (2005), pp. 1416-1420.

[2] L. Chuang, "Stochastic Petri net and system performance evaluation", Tsinghua University press, (2000), pp.19-27.

[3] Z. Yan, Z. Yong, W. Fushuan and L. Jihong, "Fuzzy fault diagnosis Petri net model is improved to accommodate the timing constraints", Automation of electric power systems, n0. 5, (2014), pp. 66-72.

[4] Z. Changyou, L. Xiaoming, Y. Yi and R. Ping, "Modeling method of workflow system oriented testing system based on Petri", Journal of University of Electronic Science and technology, no. 1, (2014), pp. 119124.

[5] Y. Yu, L. Yunyun, L. Fei and X. Qingsong, "packet north", Products Collaborative Creative design task decomposition and allocation, Journal of Chongqing University, no. 1, (2014), pp. 31-38.

[6] L. Yongrui, "the Yan army", Modeling and Simulation of raw material inventory center based on Petri net, Enterprise technology development, no. 1, (2014), pp. 13-15

[7] X. S. Halberd, M. Min and C. Dongyi, "Enhance concurrent reality game in multi task model and scheduling method", Journal of Computer Aided Design \&amp; computer graphics, no. 2, (2014), pp. 211-216.

[8] D. Zhengli, "Grid service composition based on colored Petri net", Computer science, no. 1, (2014), pp. $152-$ 155. 
[9] P. Shanliang and H. Xiting, "Research on super node model grid scheduling based on QoS", Telecom Science, no. 2, (2014), pp. 21-32.

[10] C. Jing, K. Lingfu and P. Xun, "Combined with the research on grid resource scheduling algorithm prediction mechanism and QoS constraints", Journal of computer research and development, no. 45, (2008), pp. 13-15.

[11] H. Xiaolong, "A multi-objective Petri network based on ant colony algorithm deadlock free scheduling", Electronic technology, no. 5, (2014), pp. 179-181.

[12] C. Shengyong, Z. Ruifang and H. Zhigang, "Research on grid scheduling model based on Petri net" Computer technology and automation, vol. 24, no. 4, (2012), pp. 123-125

\section{Authors}

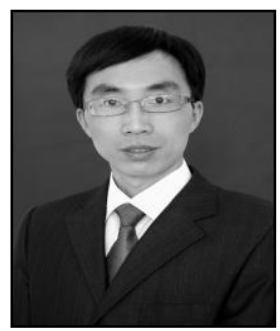

Liu Feng, he received his B.S degree in computer Science from Heilongjiang University of Science and Technology. He received his M.S degree in computer Science from Liaoning Technical University. He is an Associate professor in Heilongjiang University of Technology. His research interests include database and algorithm.

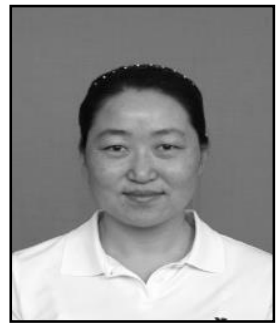

Guo WeiWei, she received her B.S degree in computer Science from Heilongjiang University of Science and Technology. She received her M.S degree in computer Science from Liaoning Technical University. She is an Associate professor in Heilongjiang University of Technology. Her research interests include database and algorithm. 